\title{
Optical Anisotropy of Quantum Disks in the External Static Magnetic Field
}

\begin{abstract}
P. SChillak ${ }^{a, *}$ AND G. CzAJKOWski ${ }^{a, b}$
${ }^{a}$ University of Technology and Life Sciences

al. S. Kaliskiego 7, 85-796 Bydgoszcz, Poland

${ }^{b}$ Scuola Normale Superiore, Piazza dei Cavalieri 7, 56126 Pisa, Italy

We show how to compute the optical functions (the complex magnetosusceptibility, dielectric function, magneto-reflection and ellipsometric spectra) for semiconductor quantum disks exposed to a uniform magnetic field in the growth direction, including the excitonic effects. The optical response is calculated for an oblique incidence of the propagating electromagnetic wave and for input waves with different polarization. The method uses the microscopic calculation of nanostructure excitonic wave functions and energy levels, and the macroscopic real density matrix approach to compute the electromagnetic fields and susceptibilities. The electron-hole screened Coulomb potential is adapted and the valence band structure is taken into account in the cylindrical approximation, thus separating light- and heavy-hole motions. The novelty of our approach is that the solution is obtained in terms of known one-particle electron and hole eigenfunctions, since, in the considered nanostructure due to confinement effects accompanied by the $\mathrm{e}-\mathrm{h}$ Coulomb interaction, the separation of the relative- and center-of-mass motion is not possible. We obtain both the eigenvalues and the eigenfunctions. The convergence of the proposed method is examined. We calculate the magnetooptical functions, including the optical Stokes parameters and ellipsometric functions for the case of oblique incidence. Numerical calculations were performed for InAs (disk)/ GaAs (barrier) disks. A good agreement with experiments was obtained.
\end{abstract}

PACS numbers: 71.35.Cc, 71.35.Ji, 73.21.La, 78.20.Ls, 78.67.Hc

\section{Introduction}

Optical properties of lower-dimensional structures are determined by the quantum size effect and excitons. In the case of excitons, the confinement of quasiparticles in a nanostructure leads to enhancement of the oscillator strength of excitons and thus to enhancement of excitonic effects. When an external magnetic field is applied, the excitonic effects are even more enhanced. Calculations

*corresponding author; e-mail: psc@utp.edu.pl 
of optical properties, taking into account all the mentioned effects (confinement, Coulomb attraction, magnetic field) are quite complicated. A separation of center-of-mass motion and the relative motion is not possible, and one has to solve a complicated boundary problem in a 6 -dimensional configuration space. Therefore various approximations have been proposed (for a review see, for example, [1] and references therein).

In the present paper we consider a quantum dot of cylindrical shape, with the symmetry axis $z$, and with infinite hard wall potentials for electrons and holes located in the $x y$ plane at the radius $R$. Such a quantum dot is also called a quantum disk (QDisk) [2]. The motivation of choosing the disk geometry can be found in Ref. [2], see also references therein. Below we compute the magnetooptical functions of QDisks when a static magnetic field $\boldsymbol{B}$ is applied in the growth direction, which is identified as the $z$-direction. As it was shown, for example, by Cortez et al. [3], the optical properties of QDisks depend on the polarization of the incident wave. In experiments by Cortez two types of waves were considered: a wave polarized in the plane of the disk (TE), a wave polarized in the growth direction $(z)$, and a TM wave obtained by a guided-wave mode. We will extend this discussion, considering the case of an oblique incidence and an arbitrary polarization of the incident wave. In addition, we will consider the effects of an applied magnetic field. The below presented calculations take into account the e-h Coulomb interaction in its full three-dimensional shape. In addition, the confinement effects and the effects of an external magnetic field are fully accounted for. The method was successfully applied in calculations of magneto- and electrooptical properties of quantum rods and superlattices $[4,5]$, and quantum disks for the case of a linearly polarized incident wave, propagating in the growth direction [6].

To obtain the optical response we apply the real density matrix approach $[7,8]$. In this approach the linear response is described by a set of coupled equations (constitutive equations (CEs)) for two-point correlation functions $\mathcal{Y}_{12 \mathrm{H} \nu}$ and $\mathcal{Y}_{12 \mathrm{~L} \nu}$ for the $\nu$-th heavy-hole exciton $(\mathrm{H} \nu)$ and the $\nu$-th light-hole exciton $(\mathrm{L} \nu)$, and a Maxwellian field equation. Below we consider the lowest H1, L1 $\rightarrow \mathrm{C}$ transition. We consider only the valence-to-conduction band transitions with the corresponding effective masses. The CEs have a form of Schrödinger's equation with a source term, which reflects the light-matter interaction. The operator $H_{\text {eh }}$, which occurs in the above equation, is the two-band exciton effective mass Hamiltonian containing kinetic energy terms, screened Coulomb interaction, confinement potentials, and the terms describing the carriers interaction with the applied magnetic field. The functions $\mathcal{Y}_{\mathrm{H}}, \mathcal{Y}_{\mathrm{L}}$ give the total polarization of our effective anisotropic medium

$$
\mathcal{P}(\boldsymbol{R}, t)=2 \int \mathrm{d}^{3} r\left[\boldsymbol{M}_{\mathrm{H}}^{*}(\boldsymbol{r}) \mathcal{Y}_{\mathrm{H}}(\boldsymbol{r}, \boldsymbol{R}, t)+\boldsymbol{M}_{\mathrm{L}}^{*}(\boldsymbol{r}) \mathcal{Y}_{\mathrm{L}}(\boldsymbol{r}, \boldsymbol{R}, t)\right],
$$

where $\boldsymbol{M}$ is the dipole density appropriate to the transition $\mathrm{H}, \mathrm{L} \rightarrow \mathrm{C}$. The above equation with the constitutive equations connect the polarization with the elec- 
tric field of the propagating wave. Both polarization and electric field must obey Maxwell's equations, which must be solved to obtain the propagation modes.

\section{Excitonic magnetosusceptibility tensor and optical functions}

We want to compute the optical linear response to a linearly polarized incident wave. The electric field $\boldsymbol{E}$ is obtained in a self-consistent way from CEs and the Maxwell equation, where the polarization (1) acts as a source. In the first step we express the functions $\mathcal{Y}$ by means of eigenfunctions $\Psi$ of the QDisk Hamiltonian. By the strong QDisk anisotropy we can separate the fast carriers movement in the plane perpendicular to the magnetic field, expanding the eigenfunction in series

$$
\begin{gathered}
\Psi\left(Z, z, \boldsymbol{\rho}_{\mathrm{e}}, \boldsymbol{\rho}_{\mathrm{h}}\right)=\sum_{n_{1}, m_{1}, n_{2}, m_{2}} \psi_{n_{1} m_{1}}^{\mathrm{e}}\left(\rho_{1}, \phi_{1}\right) \\
\times \psi_{n_{2} m_{2}}^{\mathrm{h}}\left(\rho_{2}, \phi_{2}\right) g(Z) f_{n_{1} m_{1} n_{2} m_{2}}(z)
\end{gathered}
$$

where $\psi_{n m}(\rho, \phi)$ are the known eigenfunctions of the electron (hole) motion in magnetic field taken in the symmetric gauge, and $f_{n_{1} m_{1} n_{2} m_{2}}(z)$ unknown functions to be determined; $z$ and $Z$ are the relative and the center-of-mass coordinates in the $z$-direction, respectively. Due to the disk geometry the center-of-mass motion does not influence the eigenenergies, and we assume that it practically rests at $Z=0$. The functions $\psi_{n m}(\rho, \phi)$ for a particle with charge $q$ are known and can be expressed in terms of the Kummer function of the first type, see, for example [4]. The one-particle eigenenergies $\varepsilon_{n, m}^{(\mathrm{e}, \mathrm{h})}$ follow then from the equation $\psi_{n m}(R, \phi)=0$, $R$ being the QDisk radius. The functions $f_{n m}$ and the total energy $\varepsilon$ obey an infinite system of equations

$$
\begin{aligned}
& \left(\varepsilon_{n_{1} m_{1}}^{(\mathrm{e})}+\varepsilon_{n_{2} m_{2}}^{(\mathrm{h})}-\frac{\mu_{\|}}{\mu_{z}} \frac{\partial^{2}}{\partial z^{2}}\right) f_{n_{1} m_{1} n_{2} m_{2}}(z) \\
& \quad+\sum_{n_{1}^{\prime} m_{2}^{\prime} n_{2}^{\prime} m_{2}^{\prime}} V_{n_{1} m_{1} n_{2} m_{2}^{\prime} m_{2}^{\prime} n_{2}^{\prime} m_{2}^{\prime}}^{n_{2}}(z) f_{n_{1}^{\prime} m_{1}^{\prime} n_{2}^{\prime} m_{2}^{\prime}}(z)=\varepsilon f_{n_{1} m_{1} n_{2} m_{2}}(z),
\end{aligned}
$$

$\varepsilon_{n m}$ being the corresponding one-particle eigenvalues, and $V$ the potential matrix elements

$$
\begin{aligned}
& V_{n_{1} m_{1} n_{2} m_{2}}^{n_{1}^{\prime} m_{2}^{\prime} n_{2}^{\prime} m_{2}^{\prime}}(z)=V_{i i^{\prime}}(z) \\
& \quad=\left\langle\psi_{n_{1} m_{1}}^{(\mathrm{e})} \psi_{n_{2} m_{2}}^{(\mathrm{h})}\left|\frac{-2}{\sqrt{\left(\boldsymbol{\rho}_{1}-\boldsymbol{\rho}_{2}\right)^{2}+z^{2}}}\right| \psi_{n_{1}^{\prime} m_{1}^{\prime}}^{(\mathrm{e})} \psi_{n_{2}^{\prime} m_{2}^{\prime}}^{(\mathrm{h})}\right\rangle .
\end{aligned}
$$

The functions $f_{n_{1} m_{1} n_{2} m_{2}}(z)$ must obey proper boundary conditions at the points $z= \pm\left(m_{\mathrm{e}} / \mu\right)(L / 2)$. The infinite expansion (2) is exact since the functions $\psi^{\mathrm{e}}, \psi^{\mathrm{h}}$ form a complete orthonormal set. The accuracy and convergence of the above method has been discussed in Ref. [4].

\section{Results}

Replacing the second derivative by discrete differences we transform Eqs. (3) into matrix equations which are solved numerically. The same type of equations 
hold for the heavy- and light-hole excitons. As the results we obtain the eigenenergies and eigenfunctions which are used to solve the constitutive equations. We performed numerical calculations for InAs (disk)/ GaAs (barrier) QDisks, having in mind the experimental results by Cortez et al. [3]. We used the same band parameters (effective masses, band gap, effective Rydberg and Bohr radius) as in Ref. [2]. As we noticed in Ref. [6], we can restrict the number of functions $f_{n m, n^{\prime} m^{\prime}}$ used, obtaining a good convergence. In the case considered it was enough to use six lowest order functions. The results for the three lowest energy states for heavy-hole and light-hole excitons as functions of the applied magnetic field are displayed in Fig. 1. The difference between the dotted and solid lines denotes the corresponding excitonic binding energies. Let us note the difference between the $\mathrm{H}$ and L states, both in position and curvature. Similar calculations performed for an $\mathrm{In}_{0.55} \mathrm{Al}_{0.45} \mathrm{As}$ (disk)/ $\mathrm{Al}_{0.35} \mathrm{Ga}_{0.65} \mathrm{As}$ (barrier) QDisk have shown a good agreement with the experimental results by Wang et al. [9] (see also Ref. 6).
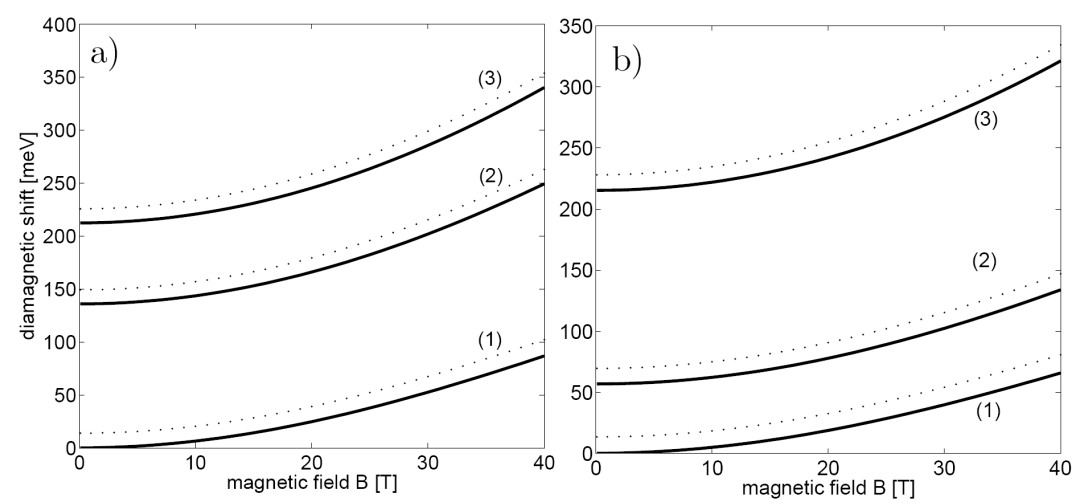

Fig. 1. (a) The calculated diamagnetic shifts of the three lowest energy states for the heavy-hole exciton in an InAs/GaAs QDisk of height $L_{z}=0.9 \mathrm{~nm}$ and an effective disk radius $R^{*}$ to fit the experiments. The value $0.4 a_{\mathrm{e}}^{*} \approx 13.9 \mathrm{~nm}$ for the disk radius gives a reasonable agreement. The dotted lines show results obtained without Coulomb $\mathrm{e}-\mathrm{h}$ interaction accounted for, and the solid lines are results including the Coulomb interaction. (b) The same for the light-hole exciton.

The so obtained eigenfunctions (2) and eigenvalues were then used to compute the heavy- and light-hole exciton amplitudes $\mathcal{Y}_{\mathrm{H}}, \mathcal{Y}_{\mathrm{L}}$, and the corresponding polarization. We assume that the QDisk material has the same band structure as the corresponding bulk material. In the case of InAs QDisks, which we will discuss, we have a direct gap, and at the $\Gamma$ point the valence band splits into the heavy-hole $\mathrm{HH}(\mathrm{H})$, and the light-hole LH $(\mathrm{L})$ subbands. The conduction band $\mathrm{C}$ will be considered as non-degenerate. An external wave with an appropriate frequency $\omega$ induces electronic transitions between the valence subbands and the conduction band. In the disk geometry we take a cylindrical approximation for 

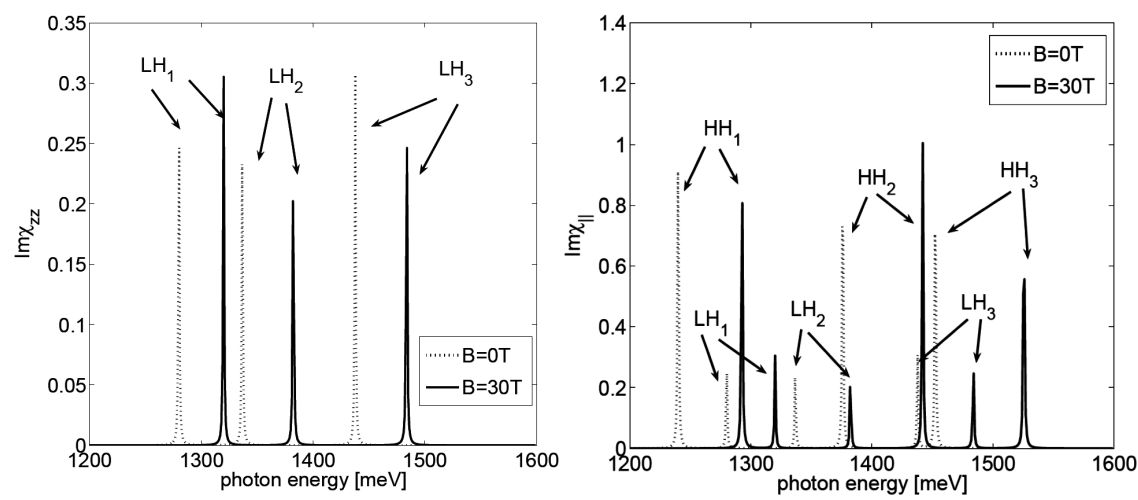

Fig. 2. The imaginary part of the QDisk susceptibility for two types of the polarization of the incoming wave.
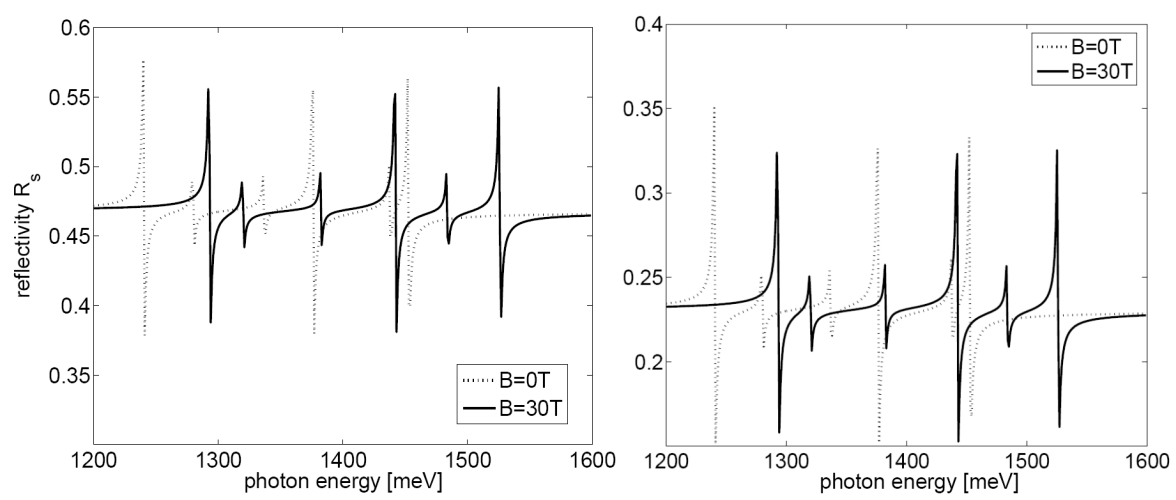

Fig. 3. The QDisk magnetoreflectance for oblique incidence, the incidence angle equals $\pi / 4$.

the bands. The transition $\mathrm{HH} \rightarrow \mathrm{C}$ is induced only by a wave polarized in plane. The transition $\mathrm{LH} \rightarrow \mathrm{C}$ is allowed for both, in-plane and $z$ polarizations (see, for example [10]). These properties are accounted for by definitions of the dipole transition moments $\boldsymbol{M}$. Assuming a point-like shape for all relevant components of $\boldsymbol{M}$, we can compute relevant components of the QDisk average complex magnetosusceptibility tensor

$$
\begin{aligned}
& \chi_{z z}(\omega, B)=\chi_{\mathrm{L}}(\omega, B), \\
& \chi_{x x}(\omega, B)=\chi_{y y}(\omega, B)=\chi_{\|}(\omega, B)=\chi_{\| \mathrm{L}}(\omega, B)+\chi_{\| \mathrm{H}}(\omega, B) .
\end{aligned}
$$

Depending on the polarization of the incoming wave, we can observe either the component $\chi_{z z}$ (z-polarization), or $\chi_{\|}$(in-plane polarization). The imaginary parts of these components for two values of the applied magnetic field are displayed in Fig. 2. We can observe a blue shift of the peaks and changes in their oscillator forces. Having the susceptibility tensor, we can determine the dielectric 
function $\underline{\underline{\epsilon}}=\epsilon_{b}+\underline{\underline{\chi}}$ and hence all the optical properties of an ensemble of quantum disks. We have computed the QDisk magnetoreflectance and the Stokes parameter $s_{3}$ (not shown). The reflectance is computed for the case of an oblique incidence where two reflection coefficients, $R_{\mathrm{s}}$ and $R_{\mathrm{p}}$ can be distinguished. They are shown in Fig. 3 for two values of the applied field.

\section{Conclusions}

We have shown how magnetooptical functions for quantum disks in the excitonic energy region can be computed with a high degree of accuracy. The structure of the optical functions spectra arises from the interplay of three types of quantization: quantization connected with the interaction of the charged particles with external magnetic field, quantization according to the Coulomb interaction and that connected with the finite size of the considered nanostructure. The dependence of the spectra on the polarization of the incoming wave is also shown.

\section{Acknowledgments}

One of us (G.C.) wishes to acknowledge the support from the grant MIURPRIN-2006/021037, and the Scuola Normale Superiore for the invitation and hospitality. We thank G. La Rocca for useful discussions.

\section{References}

[1] S. Glutsch, Excitons in Low-Dimensional Semiconductors, Theory, Numerical Methods, Applications, Springer, Berlin 2004.

[2] G. Czajkowski, L. Silvestri, CEJP 4, 254 (2006).

[3] S. Cortez, O. Krebs, P. Voisin, J.M. Gérard, Phys. Rev. B 63, 233306 (2001).

[4] P. Schillak, G. Czajkowski, Phys. Status Solidi C 5, 2495 (2008).

[5] P. Schillak, G. Czajkowski, Acta Phys. Pol. A 112, 301 (2007).

[6] P. Schillak, G. Czajkowski, to be published.

[7] A. Stahl, I. Balslev, Electrodynamics of the Semiconductor Band Edge, Springer, Berlin 1987.

[8] G. Czajkowski, F. Bassani, L. Silvestri, Rivista del Nuovo Cimento C 26, 1 (2003).

[9] P.D. Wang, J.L. Merz, S. Fafard, R. Leon, D. Leonard, G. Medeiros-Ribeiro, M. Oestreich, P.M. Petroff, K. Uchida, N. Miura, H. Akiyama, H. Sakaki, Phys. Rev. B 53, 16458 (1996).

[10] G. Bastard, Wave Mechanics Applied to Semiconductor Heterostructures, Les Editions de Physique, Paris 1989. 